\title{
Análise crítica dos diversos métodos de avaliação e registro das más oclusões
}

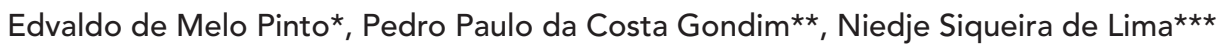

\begin{abstract}
Resumo
Desde o final do século XIX, vários sistemas de classificação tem sido propostos por diversos autores, com a finalidade, inicialmente, de facilitar o diagnóstico e o tratamento ortodôntico e, posteriormente, servir com eficácia e precisão para os estudos epidemiológicos. A classificação de Angle (1899) tornou-se o maior instrumento de medida das más oclusões durante todo o século passado. Por suas limitações, tanto na avaliação das más oclusões nos sentidos vertical e transversal como também pela falta de critérios necessários para ser considerado um instrumento epidemiológico, diversos autores propuseram outros sistemas para substituir o sistema de Angle, e para isso, realizaram testes e estudos comparativos com esses métodos e encontraram excelentes resultados. Contudo, nenhum deles foi largamente utilizado. A Organização Mundial de Saúde (OMS), a partir de 1997, passou a recomendar o uso de registro de natureza quantitativa para a avaliação de má oclusão. No futuro, novos índices deverão ser desenvolvidos e padronizados no estudo da má oclusão.
\end{abstract}

Palavras-chave: Método de registro. Má oclusão. Características oclusais.

\section{INTRODUÇÃO}

O termo má oclusão significa todos os desvios dos dentes e dos maxilares do alinhamento normal (má posição individual dos dentes, discrepância ósteo-dentária e má relação dos arcos dentais, sagital, vertical e transversal). A má relação dos arcos dentários pode refletir anormalidades nos dentes, nos maxilares ou em ambos. Para definir má oclusão, deve-se primeiro conceituar a oclusão normal. A eficiência mastigatória depende da natureza da dieta e, o alinhamento dos dentes e maxilares, não se correlaciona diretamente com a função. As características funcionais não estão usualmente presentes na distinção entre má oclusão e oclusão normal. Esta distinção é baseada no julgamento clínico da necessidade de tratamento, que não é um método bastante objetivo para uma finalidade comparativa. Estudos sobre diferenças de má oclusão na população são imprecisos devido a escassez de dados comparáveis e a ausência de padrões definidos de avaliação ${ }^{20}$.

Uma oclusão é rotulada de má oclusão com base em valores culturais (imagem do corpo e estética), desvios anatômicos de normas morfológicas, bem definidas pelo clínico, e em considerações funcionais que prejudicam a mastigação. A má

* Mestre em Ortodontia

** Mestre e Doutor em Ortodontia.

*** Especialista em ortodontia e Doutora em Odontopediatria. 
oclusão é a única, na investigação epidemiológica, que não é necessariamente compreendida como anormalidade. Muitas terapias ortodônticas são realizadas por razões estéticas ${ }^{16}$.

A Organização Mundial de Saúde, em 1962, incluiu a má oclusão sob o título "Conjunto de Anomalias Dentofaciais", as quais, são definidas como anomalias que causam deformação ou que impedem a função e que, portanto, requerem tratamento ${ }^{9}$.

Este estudo tem como objetivo, através de uma revisão de literatura, analisar os diversos métodos de registros de más oclusões existentes, bem como as características, vantagens e desvantagens do seu uso nos diversos estudos epidemiológicos.

\section{REVISÃO DE LITERATURA}

A classificação de Angle foi, provavelmente, o instrumento de medida mais utilizado no século passado e também o que sofreu mais modificações formuladas por outros autores, com a finalidade de aumentar o grau de precisão e da padronização de seus critérios. Desde então, pode-se identificar na literatura quase meia centena de índices oclusais e suas variantes.

A validade e a confiabilidade da classificação de Angle, para estudos epidemiológicos, foi questionada, pois é um indicador qualitativo e não um índice quantitativo de má oclusão. Pela classificação de Angle, as más oclusões, somente podem ser análogas (com a mesma relação oclusal) e não homólogas (com todas as características em comum $)^{4}$. Também não faz distinção entre os diferentes tipos de más oclusões que possuem relações ântero-posteriores semelhantes. Apresenta limitações por ser um sistema de discretas classes, e não um sistema de variáveis contínuas. Além disso, está restrita apenas às dimensões dentárias sagitais, não abrangendo as dimensões verticais e transversais e não levando em consideração a face ${ }^{8}$. Mesmo com as diversas críticas às suas limitações, o quase universal uso da classificação de Angle, no passado, fez dela o único indicador de prevalência dos diferen- tes tipos de má oclusão, em diferentes populações. Deve ser lembrado, também, que Angle criou a sua classificação com um objetivo de tratamento e não como uma ferramenta epidemiológica ${ }^{4,8}$.

Desde o final do século XIX, muitos pesquisadores não mediram esforços para estabelecer índices que pudessem medir a quantidade de anomalias dentofaciais existentes. Após as publicações de Angle, em 1899, e a difusão de sua classificação das más oclusões, abriu-se uma nova possibilidade de se obter uma maior precisão nestas avaliações. Contudo, os resultados obtidos, quanto a precisão, foram muito inferiores ao esperado; mesmo assim, a sua classificação continuou servindo de base a muitos métodos para a avaliação das más oclusões propostos posteriormente. Como exemplo, podemos citar os de Hellman (1921), Simon (1926), Korkhaus (1928), McCall (1944), Sclare (1945) e Moore (1949). A partir da classificação de Angle (pura ou com modificações incorporadas por outros investigadores), surgiram novas propostas e outros índices foram criados, ao longo deste período, com o objetivo de conseguir, sem êxito definitivo, uma maior precisão dos dados obtidos. Entre eles podemos mencionar os de Massler e Frankel (1951), Altemus e Van kirk (1959), HDL de Draker (1960), Comitê de especialistas da OMS (1962), Björk, Krebs e Solow (1964), HMAR de Salzman (1968), TPI de Grainger (1967), OI de Summers (1971), F.D.I. (1972), a Academia Nacional de Ciências dos EUA (1976), Slakter et al. (1980), o sistema de Ackerman-Proffitt (1988) e o IOTN de Brook e Shaw (1989). Todos foram testados com a finalidade de se obter reprodutibilidade, precisão e sensibilidade e muitos se mostraram satisfatórios para esses objetivos. Posteriormente, ainda surgiram o método proposto pela OMS (1991), o método de Katz (1992) e um novo índice proposto pela OMS, o Índice Estético Dentário (IED), em 1997, na quarta edição do manual de instruções para levantamento epidemiológico básico em saúde bucal $12,23,26,28,30$. 


\section{Métodos de registro de prevalência das más oclusões}

A má oclusão dos dentes tem sido amplamente investigada em diferentes grupos populacionais, através de vários métodos de análise e registro. Rigorosos cuidados devem ser tomados com relação à objetividade desses métodos, se diferentes estudos vão ser comparados. Tratamento ortodôntico e extrações dentárias podem eliminar uma má oclusão dependendo das circunstâncias. A maioria dos autores que realizaram estudos epidemiológicos de má oclusão enfatizou que as grandes diferenças dos resultados dos mesmos ocorreram provavelmente devido às discrepâncias metodológicas que, entre os diferentes estudos, podem ser atribuídas a dois fatores: complexidade da má oclusão e a acentuada variabilidade individual na morfologia da dentição. $\mathrm{O}$ mais importante passo para a comparabilidade de estudos epidemiológicos de má oclusão é a padronização dos critérios de registro. Registros de más oclusões tem se baseado em avaliações da morfologia da dentição e muitos diferentes procedimentos tem sido usados para esta finalidade ${ }^{12,14,21,27,28}$.

Os métodos de registros de má oclusão podem ser classificados em 4 categorias: 1 - estimativa da total freqüência de má oclusão - É a forma mais simples de registro com base em julgamento subjetivo (Se qualquer má oclusão não especificada está presente); 2 - classificação tipológica da má oclusão - (Nesta categoria está incluída a classificação de Angle). Muitos autores consideraram que as características individuais das más oclusões não foram adequadamente definidas, sendo os métodos desta categoria, relativamente subjetivos. Por isso alguns autores procuraram acrescentar novas classes, na classificação de Angle ${ }^{1}$, bem como novas características individuais na oclusão de incisivos; 3 - registro de características individuais de má oclusão - baseado na avaliação de variáveis morfológicas individuais (métodos quantitativos e qualitativos). $\mathrm{O}$ uso de métodos quantitativos está indicado para as variáveis contínuas na den- tição, ou seja, que variam, continuamente, dentro de certos limites (sobressaliência, sobremordida, oclusão molar etc.). São baseadas em medidas de cada indivíduo de um grupo estudado com a determinação de um intervalo central e de dois ou mais intervalos extremos. Os valores dos extremos são registrados como características de má oclusão. A metodologia, em princípio, não difere da utilizada nos métodos qualitativos, na qual os procedimentos técnicos são objetivos, mas, o tempo consumido em algumas medidas, dificulta a sua realização diretamente na boca. Contudo, é a mais indicada para o registro em modelos ou em radiografias cefalométricas. Os métodos qualitativos podem ser usados na avaliação de todas as variáveis morfológicas, tanto as contínuas como as discretas (número de dentes e variáveis relacionadas à erupção dentária). Uma determinada variável é analisada em relação aos critérios de registro previamente fixados. Se o critério é satisfeito, a variação é registrada como uma característica de má oclusão. Caso contrário, nenhum registro é realizado. Se os pesquisadores apresentarem o critério exato de registro das variáveis individuais, a metodologia é objetiva, e a freqüência das características pode ser comparada com o resultado de outros estudos. Se as características são citadas apenas por suas designações, como ocorre em muitos estudos, o procedimento se torna subjetivo; 4 - determinação de índices de má oclusão (sistemas de pontuação) - Tem como objetivo expressar em valores numéricos a ocorrência de determinadas características, quantitativas ou qualitativas, de má oclusão através de um sistema de pontuação (escore). Os valores são acrescentados e os índices são tratados como variáveis quantitativas. Neste sistema, para várias características são atribuídos os mesmo valores. Neste método, contudo, o registro de um grupo populacional não pode oferecer informação relativa à prevalência de determinadas manifestações de má oclusão, apenas uma medida de severidade geral de má oclusão, dentro do grupo. Existem outros métodos 
que levam em consideração os fatores etiológicos ou a indicação de tratamento ortodôntico. Neste caso, o registro de uma determinada característica morfológica depende se ela é considerada um resultado de um determinado fator etiológico, ou se nessas circunstâncias, é considerada necessária para requerer tratamento ortodôntico ${ }^{14}$.

Apesar de existirem muitos índices de registro de má oclusão, é importante distinguir os que classificam as más oclusões em tipos ${ }^{1}$, daqueles que registram prevalências em estudos epidemiológi$\cos ^{3}$ como também dos que registram a necessidade ou prioridade de tratamento ortodôntico ${ }^{7,11,25}$. Excetuando-se alguns casos, como os de diminuição da incidência de trauma nos incisivos, através da redução de suas inclinações labiais (protusão), situação que pode antecipar a necessidade de tratamento ortodôntico para uma fase mais precoce (antes dos 10 anos de idade), o mais importante benefício para os pacientes de tratamento ortodôntico pode ser a melhora estética e psicosocial, e adicionalmente os efeitos que isto pode trazer em atitudes para a saúde bucal. Isto é importante na elaboração de qualquer índice de prioridade de tratamento ${ }^{5}$. Não existe índice atual capaz de predizer o grau de problema funcional ou psicosocial dos indivíduos com relação ao estado oclusal ou deformidade facial ${ }^{29}$.

Os índices para a avaliação de alterações oclusais devem ser vistos sob dois aspectos: o do ortodontista e o do sanitarista. $\mathrm{O}$ primeiro trata de medir todos os desvios da normalidade, baseandose no número total de indivíduos que estão em desacordo com o conceito de oclusão normal e o segundo com os desvios da normalidade, relacionados à necessidade de tratamento sob o ponto de vista da adaptação do indivíduo na sociedade ${ }^{6}$. Os índices baseados em critérios puramente clínicos não satisfazem ao sanitarista e diferentes métodos têm sido utilizados com o objetivo de determinar a necessidade de tratamento ortodôntico $0^{7,11,25}$.

Em geral, os vários métodos de classificação dependem de um treinamento especial dos exa- minadores e são de caráter subjetivo. Os dados derivados destes métodos não se prestam facilmente para as análises estatísticas. Só recentemente algum esforço tem sido desenvolvido para definir o problema da má oclusão do ponto de vista da saúde pública. No passado, muitos métodos de análise e classificação foram elaborados para servir a necessidade dos ortodontistas clínicos, os quais estavam interessados no diagnóstico e nos métodos de tratamento dos casos individuais e não para servir a necessidade dos epidemiologistas ${ }^{22}$.

Os índices de má oclusão usam medidas tomadas diretamente do exame clínico do paciente ou de modelos de estudo. Exames com medidas confiáveis podem ser feitas por um examinador treinado e não necessariamente por um ortodontista. Também se evita a exposição radiográfica do indivíduo. Estes fatores são essenciais para os exames epidemiológicos em grandes grupos populacionais $^{21}$.

Grainger ${ }^{11}$, no Centro de crescimento Burlington, em Toronto, definiu o problema da má oclusão de acordo com um dos seguintes componentes: 1) estética inaceitável, 2) Significante redução na função do sistema mastigatório, 3) uma condição traumática que predispõe a destruição tecidual na forma de doença periodontal ou cárie, 4) Problemas na fala, 5) Falta de estabilidade na oclusão e 6) anomalias congênitas ou traumas. Baseado nestas definições, e usando uma amostra populacional e avaliações dos ortodontistas, desenvolveu um importante índice para priorizar a necessidade de tratamento: O Índice de Prioridade de Tratamento (Treatment Priority Índex - TPI), que usa equações de regressão para sintetizar componentes de má oclusão, dentro de uma pontuação (escore) de 0 à $10+$, que indica a relativa severidade da má oclusão. Estes componentes incluem: 1) Relação de primeiro molar (neutro, mesio e disto oclusão), 2) Relação horizontal dos incisivos (sobressaliência), 3) Relação vertical dos incisivos (sobremordida), 4) Deslocamentos dentários (apinhamentos e rotações), 5) Ausência congênita de dentes e 6) 
Mordida cruzada posterior. A interpretação proposta pelos escores foi $0=$ normal, $1-3=$ desvios menores (nenhum tratamento indicado), 4-6 = desvios definidos (tratamento eletivo), 7-9 = problema de má oclusão (tratamento desejável) e 10+ = problema severo de má oclusão (tratamento necessário). Foi usado muito pouco, nos estudos epidemiológicos, por ser considerado subjetivo, isto é, utilizando o julgamento clínico para a indicação da necessidade de tratamento. Com relação aos problemas sagitais de má oclusão, este método avalia a relação antero-posterior entre os maxilares com base na oclusão dos molares e caninos, classificando os casos em neutro, disto e mesio oclusão ${ }^{13}$.

O índice proposto por Draker ${ }^{7}$, o Problema de Desvios Lábioslinguais (Handicapping Labiolingual Deviations - HLD), foi uma tentativa de se obter um método para complementar e, talvez, substituir o julgamento clínico que é vulnerável pela sua subjetividade. $\mathrm{O}$ que é necessário para os cirurgiões-dentistas da área de saúde pública, portanto, é um instrumento administrativo, tal como um índice. Em contrapartida, o ortodontista requer um instrumento de classificação e diagnóstico. A diferença entre essas duas visões foi importante para as considerações deste trabalho. Este índice foi submetido aos seguintes testes: 1) Simplicidade (tempo requerido), 2) Reprodutibilidade e 3) Validade, mostrando excelentes resultados em todos eles ${ }^{7}$.

O método de Björk, Krebs e Sollow ${ }^{3}$ consiste em um método de registro sistemático, qualitativo, de todas as características morfológicas individuais de uma má oclusão, separadamente ou em combinação. Estas características estão reunidas em três principais grupos: 1) Anomalias na dentadura (anomalias dentárias, erupção anormal e desalinhamento dentário), 2) Anomalias oclusais (desvios na relação entre os arcos dentais superior e inferior) e 3) Desvios na condição de espaço (diastema ou apinhamento de dentes). Os dados são registrados em código e uma indicação de necessidade de tratamento está incluída no registro.
Os casos de múltiplas extrações, cáries extensas, severas condições patológicas e os que fizeram ou estão fazendo tratamento ortodôntico são excluídos do registro de más oclusões ${ }^{3}$.

Howitt, Stricket e Henderson ${ }^{15}$ desenvolveram um Índice estético, o Eastman Esthetic Index, para medir a satisfação do paciente com relação a sua boca e os seus dentes. Katz ${ }^{17}$ em uma revisão de oito índices ortodônticos, encontrou que todos mostraram alguma capacidade de distinguir indivíduos que estavam satisfeitos com a sua aparência bucal daqueles que não estavam. Eles encontraram, contudo, que esta capacidade foi fraca e que a classificação de Angle mostrou uma capacidade mais forte e consistente para distinguir os dois grupos. Concluiram que a classificação de Angle melhor representa a relação de perfil para o paciente $^{15,17}$.

O método proposto por Salzmann ${ }^{25}$ e aprovado pela American Dental Association e pela American Association of Orthodontists, o Registro de Avaliação de Problemas de Má Oclusão (Handicapping Malocclusion Assessment Record - HMAR), teve como objetivo estabelecer uma média de prioridade de tratamento de problemas de má oclusão nas crianças, de acordo com a severidade mostrada pela magnitude da pontuação (escore) obtida na análise de má oclusão de modelos dentários ou diretamente na cavidade bucal.

Outros índices baseados nas relações dentárias são o Índice oclusal (Occlusal Index - OI) de Summer (1971) e o Método Uniforme para Análise Epidemiológica de Má Oclusão (Uniform Methods for the Epidemiologic Assessment of Malocclusion) desenvolvido pela Organização Mundial de Saúde (1974). Nenhum destes métodos foi largamente empregado ${ }^{21}$.

Baume $^{2}$ relatou que desde 1962 a Organização Mundial de Saúde (OMS) preconiza levantamentos epidemiológicos da condição oclusal através de índices simples e de fácil execução, cujo propósito é permitir a geração de um volume maior de dados comparáveis em nível mundial e lograr 
informações sobre aspectos genéticos e sociais de certas oclusopatias. Mesmo tendo elaborado e modificado um índice próprio de classificação da má oclusão, mas reconhecendo a falta de consenso entre os pesquisadores em relação à aceitação de um índice oclusal, ela sugeriu, em 1977, que um índice oclusal poderia ser selecionado de acordo com as características sanitárias, econômicas e políticas de uma determinada região. $\mathrm{Na}$ terceira edição do manual de instruções para levantamento epidemiológico (1991), apresentou uma metodologia para estudos populacionais, codificando e caracterizando as condições oclusais do seguinte modo: código $0=$ Normal (ausência de alterações oclusais), Código 1 = Leve (um ou mais dentes com giroversão ou ligeiro apinhamento ou espaçamento prejudicando o alinhamento regular) e Código 2 = Moderada/Severa (quando há um efeito inaceitável sobre a aparência facial ou uma significativa redução da função mastigatória ou problemas fonéticos). A Faculdade de Saúde Publica da USP (FSP-USP), conforme consta no anteprojeto do Ministério da Saúde para levantamento das condições bucais em todo o Brasil - SB2000 - (Ministério da Saúde, 1999), fez algumas alterações a essa metodologia, recomendando incluir na condição leve (código 1), os casos não explicitados nos critérios descritos pela OMS, tais como mordida cruzada posterior e sobremordida ou transpasse vertical acima de $2 \mathrm{~mm}$. A partir de 1997, uma mudança acentuada é impressa às recomendações da OMS com relação à mensuração de má oclusão e, se antes era preconizado um registro de natureza qualitativa, passou a ser recomendado o uso de um registro de natureza quantitativa ${ }^{2}$.

\section{Estudos comparativos realizados para testar a confiabilidade entre os diversos métodos de avaliação da má oclusão}

Gravely e Johnson ${ }^{12}$ realizaram uma pesquisa para testar a confiabilidade da classificação de Angle, em duas amostras da faculdade de Odontologia da Universidade de Leeds, Gran Bretanha.
Uma de 102 pares de modelos de estudo de prétratamento de pacientes tratados ortodonticamente, na fase de dentadura mista e permanente, e outra de 80 crianças escolares, com idades de 11 a 15 anos, selecionadas para tratamento ortodôntico. As más oclusões foram classificadas por três experientes ortodontistas e foram realizados exames intra e interexaminadores. Nos exames em modelos de estudo, as más oclusões de classe III e de classe II divisão 1 foram as categorias mais confiáveis com percentuais de concordância variando entre $86-95 \%$ para a classe III e $85-88 \%$ para a classe II divisão 1 . O nível de concordância para a classe I foi menor, variando entre 66$75 \%$. Para a classe II divisão 2 foi menor ainda, com uma variação de 47-67\%. Nos exames feitos diretamente nas crianças, as categorias classe I e classe II divisão 1 mostraram um nível de confiabilidade similar ao encontrado nos modelos de estudo, $75-78 \%$ para a classe I e $76-90 \%$ para a classe II divisão 1. As categorias classe II divisão 2 e classe III mostraram maiores diferenças entre os três examinadores, embora os números tenham sido considerados pequenos para validar conclusões. Nivel elevado de erros intra-examinadores foram encontrados, bem como foi observado alto nível de erro inter-examinadores, principalmente na categoria classe II divisão 2. O grupo de maior consistência classificado em ambos os estudos foi a classe II divisão 1. Concluíram que a classificação das más oclusões podem ser feitas pelo exame clínico com um nível de confiabilidade similar ao da classificação em modelos de estudos e que o sistema de Angle não pode ser utilizado com um alto nível de confiabilidade, podendo ser, portanto, um confiável método de classificação de má oclusão em estudos em comunidades, contanto que os exames, em diferentes comunidades, sejam feitos pelo mesmo examinador.

$\mathrm{Katz}^{18}$ distribuiu um questionário com fotografias de dez modelos de estudo (de gesso), em oclusão, três com oclusões ideais e sete com vários graus de classe II, para 347 ortodontistas especia- 
lizados em 1980 ou há mais tempo. Houve 77,8\% de respostas. O resultado mostrou uma discordância de $50 \%$ para três das dez fotografias de modelos. Em uma tentativa de oferecer uma solução para o debate sobre a classificação de Angle, propôs um método de classificação que mantivesse a nomenclatura de Angle mas que mudasse algumas regras no seu sistema de medidas. Uma mudança feita por Katz ${ }^{18}$ foi usar os pré-molares como os dentes de referência, mais do que os primeiros molares, dentes escolhidos como referência por Angle $^{1}$. Posteriormente, ao contrário de Angle, o sistema de $\mathrm{Katz}^{18}$ foi adaptado para as dentaduras decídua e mista, tão bem como aplicado nos casos ortodônticos onde pré-molares foram extraídos. De acordo com a sua classificação, a classe I foi definida como a distância entre o meio da cúspide do pré-molar superior mais anterior e a ameia criada pelo contato distal do pré-molar inferior mais anterior. A relação ideal foi designada por Katz como 0 (zero), enquanto o sinal mais (+) indica uma direção de classe II e o sinal menos (-) aponta para uma tendência de classe III. A mesma regra foi aplicável para o primeiro molar decíduo nas dentaduras decídua e mista. Cada lado da dentadura é avaliado separadamente.

$\mathrm{Du}$, Rinchuse e Zullo ${ }^{8}$ realizaram um estudo com o objetivo de testar a confiabilidade da classificação de Angle e comparar os resultados com os de outros dois sistemas, o de Katz e o Sistema Britânico de Classificação de Incisivos. Para a classificação de Angle encontraram que os valores de confiabilidade interexaminador foram muito menores do que os de intra-examinador. Grandes diferenças ocorreram no nível de concordância entre os examinadores. Somente dois dos vinte e cinco casos avaliados receberam a mesma classificação de todos os quatro examinadores. Dezesseis modelos foram concordantes para serem classe III e vinte e um modelos para serem classe II, divisão 2. Nove modelos tinham menos que três concordâncias de examinadores em sua classificação. Desses, cinco mostraram discordâncias entre as seguintes clas- ses de Angle: classe I, classe II divisão 1 e classe II divisão 1 subdivisão. Para a classificação britânica de incisivos, a confiabilidade interexaminador foi menor do que a intra-examinador. Oito dos vinte e cinco modelos receberam a mesma classificação de todos os quatro examinadores nas duas sessões de exame (quatro classe III e quatro classe II divisão 1). Três modelos tinham menos do que três examinadores concordantes em suas classificações. Para a classificação de Katz, os valores de confiabilidade interexaminadores foram relativamente mais consistentes do que os intra-examinadores, contrastando com os resultados encontrados nas outras duas classificações. A confiabilidade intraexaminador foi maior do que a interexaminador em ambas as classificações, Angle e britânica, sendo a confiabilidade interexaminador maior na britânica do que na de Angle. A classificação de Katz foi a mais precisa de todas, enquanto a classificação de Angle foi a de menor confiabilidade dos três sistemas estudados. Concluiu que os casos selecionados foram mais difíceis de classificar e os resultados devem ser diferentes se as mesmas classificações forem usadas para classificar casos mais comuns, típicos, do dia a dia. Recomendou atenção para o uso da classificação de Angle, pelas suas limitações e fragilidades. O sistema de Katz parece ser mais indicado para pesquisa pela sua natureza contínua e deve ser usado em estudos epidemiológicos.

Brin, Weinberger e Ben-Choirin ${ }^{4}$ realizaram um estudo com o objetivo de comparar o percentual de concordância de um sistema combinado dos métodos de avaliação de sobressaliência e sobremordida preconizados por William e Stephens, em 1992, e o método de Katz ${ }^{18}$ e averiguar se o sistema combinado seria mais satisfatório do que o de Angle ou o de Katz, separadamente. O objetivo da combinação dos dois métodos foi que a adição numérica permitiria uma classificação mais válida. Os resultados mostraram que os três métodos de classificação, de acordo com o seu percentual de concordância, tiveram a seguinte seqüência na ordem descendente: método modificado de Katz, 
o sistema combinado e a classificação de Angle. Esta seqüência foi a mesma para ambos os grupos de examinadores, ortodontistas qualificados e estudantes. No sistema combinado, o percentual de concordância foi significantemente reduzido. Com relação ao grau de experiência profissional dos examinadores, o nível de desempenho dos estudantes foi abaixo dos ortodontistas, quando foi utilizado o método de Angle, sendo a diferença estatisticamente significante. Entretanto, isso não foi observado entre esses dois grupos para os outros dois métodos, o de Katz e o combinado. Os valores de Kappa para os métodos de Angle e de Katz indicaram resultados pobre e bom, respectivamente. As características incorporadas ao método modificado de Katz são satisfatórias para o objetivo de ensino, embora somente como uma classificação suplementar. A técnica combinada utilizada neste estudo não mostrou nenhuma vantagem sobre ambos os métodos, a classificação clássica de Angle e a técnica modificada de Katz, não sendo recomendada a sua aplicação clínica. Uma análise mais aprofundada nos resultados obtidos neste estudo revelou que, em um determinado número de casos, o método de Angle produziu um percentual igualmente menor de concordância para os ortodontistas e para os estudantes. Alguns casos demonstraram, portanto, uma aplicabilidade limitada para o método de Angle. Estes mesmos casos, quando classificados por ambos os métodos, o modificado de Katz e o combinado, apresentaram um percentual de concordância acentuadamente maior em ambos os grupos de examinadores. O quantitativo método modificado de Katz supera o problema de julgamento por introduzir um sistema contínuo de medida. A confiabilidade do método modificado de Katz foi demonstrada pelo alto valor de Kappa e pelo percentual de concordância.

\section{DISCUSSÃO}

A classificação de Angle foi um importante passo no desenvolvimento da Ortodontia. Não só subdividiu a maioria dos tipos de má oclusão, mas também forneceu a primeira clara definição de oclusão normal na dentadura natural. Apesar de ser alvo de críticas, ela tem permanecido largamente aceita e usada na maioria das Faculdades de Odontologia. Um grande número de classificações e índices tem sido desenvolvidos mas nenhum deles foi, ainda, universalmente aceito e isto ocorre devido às variações na terminologia, diferenças de idade e sexo nas amostras e níveis de rigor e precisão dos métodos de exame ${ }^{24}$.

A multiplicidade de sistemas de análises de registro de má oclusão indica, por si mesmo, a dificuldade de se encontrar um método que satisfaça o serviço comunitário ortodôntico e ao mesmo tempo seja prático e confiável na sua aplicação. Dos métodos já abordados, apenas os de Björk, Krebs e Solow ${ }^{3}$ e o da FDI $^{5}$ medem e registram fatores oclusais ${ }^{3}$. Todos os outros métodos usam suas medidas de fatores oclusais para atender algum outro propósito. Grainger, Salzmann e Summers utilizam os vários escores de desvio das características de oclusão ideal para determinar um sistema de pontuação (escore) de severidade de má oclusão ${ }^{10}$.

Um dos maiores problemas na avaliação de má oclusão é a escolha de um índice que seja confiável e reprodutível. Em uma tentativa de distinguir pacientes com necessidades de cuidados ortodônticos, vários sistemas de classificação de má oclusão tem sido propostos. Índices de tratamento ortodôntico tentam quantificar a aparência e servem como instrumentos epidemiológicos para a análise da prevalência de má oclusão ou mudanças em seu padrão de doença. Características morfológicas de má oclusão, quando avaliadas à luz da informação psicosocial, permitem aos epidemiologistas projetar necessidades nacionais de tratamento ${ }^{19}$.

A alta prevalência de má oclusão encontrada na maioria destes estudos tem levado alguns investigadores a abandonar o conceito de má oclusão. Critérios e métodos de exame padronizados para todas as doenças e desordens bucais estão agora sendo desenvolvidos. Existe uma concordância 
generalizada que a má oclusão tem uma etiologia multifatorial e que as duas categorias básicas são genética e ambiental. Algum progresso adicional no estudo epidemiológico de anomalias oclusais possibilitará o desenvolvimento de um índice satisfatório. $\mathrm{O}$ rápido desenvolvimento dos estudos epidemiológicos da cárie dentária, durante os últimos 30 anos, dependeu de uma quase universal aceitação do DMF como um índice. Em termos de sua etiologia e manifestações, o estudo da má oclusão parece ser muito mais complexo do que o da cárie. As alterações oclusais não constituem uma variável discreta do tipo presente ou ausente e podem ser encontradas em uma quantidade contínua de situações, da saúde à incapacidade. A procura de um índice baseado em uma objetividade absoluta, talvez, seja um procedimento inconsistente. Nenhum dos índices desenvolvidos desde 1951 tem sido usado em larga escala nas investigações epidemiológicas. Também devem ser incorporados a eles alguns elementos subjetivos, como a percepção e os valores das pessoas afetadas pela má oclusão, que são as mais favorecidas pelos estudos epidemiológicos ${ }^{16}$.

Apesar do conhecimento sobre a oclusão ter evoluído enormemente no decorrer do século XX, ainda não foi proposta uma forma de classificação oclusal que pudesse ser adotada como padrão ${ }^{28}$.

\section{CONCLUSÕES}

Com base na literatura revisada, conclui-se que:

1) estudos comparativos e testes foram realizados com a classificação de Angle e mostraram pouca confiabilidade no seu uso em estudos epidemiológicos.

2) a Organização Mundial de Saúde (OMS), na quarta edição do seu manual de instruções para levantamentos epidemiológicos (1997), passou a recomendar o uso de um registro de natureza quantitativa para a avaliação das más oclusões.

3) mesmo com todo o conhecimento adquirido sobre a oclusão ainda não foi proposta uma forma de classificação e registro que pudesse ser adotada como padrão para os estudos de má oclusão.

4) é necessário o desenvolvimento de novos índices ou uma modificação naqueles já existentes, com a finalidade de conciliar a confiabilidade e reprodutibilidade em um método simplificado e rápido, para se obter, então, uma padronização nos métodos de avaliação das más oclusões.

Enviado em: fevereiro de 2006 Revisado e aceito: julho de 2006

\title{
Critical analyses of some malocclusions register and evaluation methods
}

\begin{abstract}
Since de end of century $20^{\text {th }}$, a number of classification systems have being proposed, by several authors, with the intention of, initially, facilitate the diagnosis and the orthodontic treatment, and later, be of efficiency and precision to the epidemiological studies. The Angle's classification (1899) became the measurements greater instrument of malocclusions during all last century. Because of its limitations, as much in the evaluation of malocclusions in vertical a transversal planes as for the criterion lack needed to be consider as an epidemiological instrument, many authors proposed others systems to replace the Angle's system, and to do so, the carried out many tests and comparatives studies with these methods and found optimal results. However, none of them was largely used. The World Health Organization (WHO), from 1997, started to recommend the use of quantitative nature registers to evaluate malocclusions. In the future, new index will be developed and standardized in the malocclusion study.
\end{abstract}

Key words: Register Method. Malocclusion. Oclusal characteristics. 


\section{REFERÊNCIAS}

1. ANGLE, E. H. Classification of Malocclusion. Dental Cosmos Philadelphia, v. 41, no. 3, p. 248-264, Mar. 1899

2. BAUME, L. J. Uniform methods for the epidemiologic assessment of Malocclusion. Am. J. Orthod., St. Louis, v. 66, no. 3, p. 251-272, Sept. 1974.

3. BJORK, A.; KREBS, A.; SOLOW, B. A Method for Epidemiologic Registration of Malocclusion. Acta Odont. Scand., Stock Holmes, v. 22, no. 1, p. 27-41, Feb. 1964.

4. BRIN, I.; WEINBERGER, T.; BEN-CHOIRIN, E. Classifications of occlusion reconsidered. Eur. J. Orthod., Oxford, v. 22, no. 2, p.169-174, 2000.

5. BROK, P. H.; SHAW, W. C. The development of an index of orthodontic treatment priority. Eur. J. Orthod., Oxford, v. 11, no. 3, p. 309-320, Aug. 1989.

6. CHAVES, M. M. Manual de Odontologia Sanitária. São Paulo: Massao Ohno, 1960. t. 1.

7. DRAKER, H. L. Handicapping labio-lingual desviations: a proposed index for public health purposes. Am. J. Orthod., St. Louis, v. 46, no. 4, p. 295-305, Apr. 1960.

8. DU, S. Q.; RINCHUSE, D. J.; ZULLO, T. G. Reliability of three methods of occlusion classification. Am. J. Dentofacial Orthop., St. Louis, v. 113, no. 4, p. 463-470, 1998.

9. FOSTER, T. D.; DAY, A. J. W. A survey of malocclusion and the need for Orthodontic treatment in a Shropshire school population. Br. J. Orthod., Oxford, v. 1, no. 3, p. 73-78, 1974.

10. ; MENEZES, D. M. The assessment of occlusal features for public health planning purposes. Am. J. Orthod., St. Louis, v. 69 , no. 1, p. 83-90, 1976.

11. GRAINGER, R. M. Orthodontic treatment priority index. Vital Health Stat 2, Rockville, no.25, p.1-49, Dec. 1967.

12. GRAVELY, J. F.; JOHNSON, D. B. Angle's Classification of Malocclusion: an assessment of reliability. Br. J. Orthod., Oxford, v. 1, no. 3, p. 79-86, Apr. 1974.

13. GRECCO, A. J. T. Contribuição para o estudo da prevalência de anormalidades da oclusão em escolares do município de Araraquara, amarelos nascidos no Japão, amarelos nascidos no Brasil (Niseis) e brancos nascidos no Brasil, sua relações com a higiene oral e as gengivites. 1966. $77 \mathrm{f}$. Tese (Doutorado)-Faculdade de Farmácia e Odontologia de Araraquara, Araraquara, 1966.

14. HELM, S. Prevalence of malocclusion in relation to development of the dentition. An epidemiological study of Danish school children. Acta Odontol. Scand., p. 9-117, June 1970. supplementum 58.

15. HOWITT, J. W.; STRICKER, G.; HENDERSON, R. Eastman esthetic index. N. Y. State Dent. J., Albany, v. 33, no. 4, p. 215-220, Apr. 1967.

16. JAGO, J. The epidemiology of dental occlusion: a critical appraisal. J. Public Health Dent., Raleigh, v. 34, no. 2, p. 80-93, Spring 1974
17. KATZ, R. V. Relationships between eight Orthodontics indices and an oral self-image satisfaction scale. Am. J. Orthod., St. Louis, v. 73, p. 328-334, 1978.

18. KATZ, M. I. Angle classification revisited 2: a modified Angle classification. Am. J. Dentofacial Orthop., St. Louis, v. 102, p. 277-284, 1992.

19. LEW, K. K.; FOONG, W. C.; LOH, E. Malocclusion prevalence in an ethnic Chinese population. Aust. Dent. J., Sydney, v. 38 no. 6, p. 442-449, Dec. 1993.

20. LOMBARDI, A. V.; BAILIT, H. L. Malocclusion in the Kwaio, a Melanesian group on Malaita, Solomon Islands. Am. J. Phys. Anthropol., New York, v. 36, no. 2, p. 283-293, Mar. 1972.

21. MC LAIN, J. B.; PROFFIT, W. R. Oral Health Status in the United States: prevalence of malocclusion. J. Dent. Educ., Washington, D.C., v. 49, no. 6, p. 386-397, June 1985.

22. MASSLER, M.; FRANKEL, J. M. Prevalence of Malocclusion in children aged 14 to 18 years. Am. J. Orthod., St. Louis, v. 37, no. 10, p. 751-768, Oct. 1951.

23. MOYERS, R. E. Classificação e terminologia da maloclusão. In: Ortodontia. 4. ed. Rio de Janeiro: Guanabara Koogan, 1991. cap. 9, p. 156-166.

24. SALEH, F. K. Prevalence of Malocclusion in a Sample of Lebanese Schoolchildren: an epidemiological study. East Mediterr. Health J., Alexandria, v. 5, no. 2, p. 337-343, Mar. 1999.

25. SALZMANN, J. A. A handcapping malocclusion assessment to establish treatment priority. Am. J. Orthod., St. Louis, v. 54, no. 1, p. 749-765, Oct. 1968.

26. SATURNO, L. D. Características de la oclusión de 3630 escolares del área metropolitana de Caracas. Acta Odontol. Venez., Caracas, v. 18, n. 2, p. 237-263, mayo/ago. 1980.

27. SILVA, R. G.; KANG, D. S. Prevalence of Malocclusion among Latino adolescents. Am. J. Orthod. Dentofacial Orthop., St. Louis, v. 119, no. 3, p. 313-315, Mar. 2001.

28. STIZ, A. L. Prevalência da doença periodontal e da má oclusão dentária em escolares de 5 a 12 anos de idade de Camboriú-SC, 2000. 2001. 74 f. Dissertação (Mestrado)Faculdade de Saúde Pública, Universidade de São Paulo, São Paulo, 2001.

29. STRICKER, D. E.; CLIFFORD, L. K.; COHEN, D. B.; GIDDON, L. H.; EVANS, C. A. Psychosocial aspects of craniofacial disfigurement. A "State of the Art" assessment conducted by the craniofacial anomalies program branch, The National Institute of Dental Research. Am. J. Orthod., St. Louis, v. 76, no. 4, p. 410-422, 1979.

30. TANG, E. L. K.; WEI, S. H. Y. Recording and measuring malocclusion: a review of the literature. Am. J. Orthod. Dentofacial Orthop., St. Louis, v. 103, no. 4, p. 344-351, Apr. 1993.
Endereço para correspondência

Edvaldo de Melo Pinto

Av. Domingos Ferreira, 2160/706 - Boa Viagem

CEP: 51.111-020 - Recife - PE

E-mail: edvaldopinto@terra.com.br 